\title{
Genetic analysis of phytoene synthase 1 (Psy1) gene function and regulation in common wheat
}

\author{
Shengnan Zhai ${ }^{1}$, Genying Li ${ }^{2}$, Youwei Sun ${ }^{1}$, Jianmin Song ${ }^{2}$, Jihu Li ${ }^{1}$, Guoqi Song ${ }^{2}$, Yulian Li ${ }^{2}$, Hongqing Ling ${ }^{3}$, \\ Zhonghu $\mathrm{He}^{1,4^{*}}$ and Xianchun $\mathrm{Xia}^{1{ }^{*}}$ (D)
}

\begin{abstract}
Background: Phytoene synthase 1 (PSY1) is the most important regulatory enzyme in carotenoid biosynthesis, whereas its function is hardly known in common wheat. The aims of the present study were to investigate Psy? function and genetic regulation using reverse genetics approaches.

Results: Transcript levels of Psy 1 in RNAi transgenic lines were decreased by $54-76 \%$ and yellow pigment content (YPC) was reduced by 26-35\% compared with controls, confirming the impact of Psy1 on carotenoid accumulation. A series of candidate genes involved in secondary metabolic pathways and core metabolic processes responded to Psy1 down-regulation. The aspartate rich domain (DXXXD) was important for PSY1 function, and conserved nucleotides adjacent to the domain influenced YPC by regulating gene expression, enzyme activity or alternative splicing. Compensatory responses analysis indicated that three Psy 1 homoeologs may be coordinately regulated under normal conditions, but separately regulated under stress. The period 14 days post anthesis (DPA) was found to be a key regulation node during grain development.
\end{abstract}

Conclusion: The findings define key aspects of flour color regulation in wheat and facilitate the genetic improvement of wheat quality targeting color/nutritional specifications required for specific end products.

Keywords: Carotenoid biosynthesis, RNAi, RNA-Seq, TILLING, Triticum aestivum

\section{Background}

Carotenoids, a complex class of C40 isoprenoid pigments synthesized by photosynthetic organisms, bacteria and fungi [1], are essential components of the human diet. The most important function is as a dietary source of provitamin A [2]. Vitamin A deficiency can result in xerophthalmia, increased infant morbidity and mortality, and depressed immunological responses [3]. Additionally, carotenoids as antioxidants can reduce the risk of agerelated macular degeneration, cancer, cardiovascular diseases and other chronic diseases [4]. Common wheat (Triticum aestivum L.) is a major cereal crop, supplying significant amounts of dietary carbohydrate and protein for over $60 \%$ of the world population. It is also an

\footnotetext{
* Correspondence: zhhecaas@163.com; xiaxianchun@caas.cn

'Institute of Crop Science, National Wheat Improvement Center, Chinese Academy of Agricultural Sciences (CAAS), 12 Zhongguancun South Street, Beijing 100081, China

Full list of author information is available at the end of the article
}

important source of carotenoids in human diets [5]. Moreover, carotenoids in wheat grains determine flour color, an important quality trait for major wheat products such as noodles.

Phytoene synthase (PSY) catalyzes a vital step in carotenoid biosynthesis, generally recognized as the most important regulatory enzyme in the pathway $[1,6]$. Although there are up to three PSY isozymes in grasses, only Psy1 expression is associated with carotenoid accumulation in grains $[7,8]$. The wheat Psy1 gene was cloned based on the sequence homology, and QTL analysis showed that Psy1 co-segregated with yellow pigment content (YPC), which is significantly related to carotenoids $(r=0.8)[6,9]$. To date, several studies have focused on homology-based cloning of Psy1 and QTL analysis, whereas gene function and regulation remain to be determined. 
Common wheat has a large genome that consists of three closely related (homoeologous) genomes with 93$96 \%$ sequence identity and a high proportion of repetitive sequences $(>80 \%)$ [10]. Homoeologous gene duplication limits the use of forward genetics due to compensatory processes that mask the effects of single-gene knockout mutations [11]. Therefore, the ability to investigate gene function and regulation in wheat ultimately depends on robust, flexible, high-throughput reverse genetics tools.

RNA interference (RNAi) is a sequence-specific gene suppression system that has been used in a variety of plant species as an efficient tool to decrease or knockout gene expression. RNAi has an enormous potential in functional genomics of common wheat, because all homoeologs (from the A, B and D subgenomes) can be simultaneously silenced by a single RNAi construct [12]. To date, RNAi has been used to target a wide range of genes in wheat, including those encoding lipoxygenase, starch biosynthetic enzymes, and proteins involved in storage [13-15].

With next-generation high-throughput sequencing technologies, RNA-sequencing (RNA-Seq) has emerged as a useful tool to profile genome-wide transcriptional patterns in different tissues and developmental stages, and can lead to the discovery novel genes in specific biological processes [16]. In this context, comparative analysis of transcriptome data between transgenic lines and wild type can reveal the transcriptional regulation network associated with genetic change.

Targeting induced local lesions in genomes (TILLING) is a powerful reverse genetics approach combining chemical mutagenesis with a high-throughput screen for mutations, and has been widely used in functional genomics [17]. Compared to typical reverse genetics techniques such as RNAi and insertional mutagenesis, the main advantage of TILLING is the ability to accumulate a series of mutated alleles, including silent, missense, truncation or splice site changes, with a range of modified functions, from wild type to almost complete loss of function [17]. These mutations are excellent materials for understanding gene function, genetic regulation and compensatory processes [18]. Moreover, alleles generated by TILLING can be used in traditional breeding programs since the technology is non-transgenic and the mutations are stably inherited.

The main objectives of the present work were to investigate Psy1 function and genetic regulation using three complementary reverse genetics approaches. Psy1 was specifically silenced in wheat grain by RNAi to confirm Psy1 function. Comparative analysis of transcriptome data between transgenic lines and non-transformed controls by RNA-Seq was used to reveal the transcriptional regulation network responding to Psy1 down-regulation. In addition, two EMS (ethyl methanesulfonate)-mutagenised wheat populations were screened for mutations in Psy1 by TILLING to obtain a series of Psy1 alleles with potential to increase our understanding of the gene function, genetic regulation and compensatory processes. This integrative approach provided new insights into the molecular basis and regulatory processes of carotenoid biosynthesis in wheat grain.

\section{Methods}

\section{Wheat transformation and regeneration}

The binary vector pSAABx17 containing the endospermspecific promoter of HMW-GS (High-Molecular-Weight Glutenin Subunits) Bx17, the nopaline synthase (Nos) terminator, and a selectable neomycin phosphotransferase II (npt II) gene, was used to construct an RNAi vector. The first exon of Psy1 (EF600063; $460 \mathrm{bp)} \mathrm{was} \mathrm{selected} \mathrm{as}$ the trigger fragment. Briefly, the sense fragment of Psy1 was amplified using the primer pair PS-F containing a BamHI site and PS-R with an AsuII site, while the antisense fragment was amplified with primers PA-F containing a KpnI site and PA-R including a NheI site (Additional file 1: Table S1). The fourth intron of Psy1 as the spacer was amplified by primers In-F and In-R. All sequences and directions of the inserts were confirmed by sequencing. The final RNAi construct was named pRNAiPsy1 (Fig. 1).

pRNAiPsy1 was transformed into wheat cultivar NB1 by Agrobacterium tumefaciens-mediated transformation [19]. Briefly, immature seeds were collected at 14 DPA and sterilized with $70 \%$ ethanol for $1 \mathrm{~min}, 20 \%$ bleach for $15 \mathrm{~min}$ and rinsed three times with sterile water. Isolated immature embryos were precultured on the induction medium for $4 \mathrm{~d}$ in dark at $25{ }^{\circ} \mathrm{C}$. Then, the embryos were inoculated with a drop of $A$. tumefaciens suspension and co-cultured for $3 \mathrm{~d}$ on the same medium. The immature embryos were cultured on selection medium at $25{ }^{\circ} \mathrm{C}$ in the dark for 3 weeks for callus induction. Then, the calli were transferred onto regeneration medium at $25{ }^{\circ} \mathrm{C}$ in the light with a density of $45 \mu \mathrm{mol} \mathrm{m} \mathrm{m}^{-2} \mathrm{~s}^{-1}$ and $16 \mathrm{~h}$ photoperiod for another 3 weeks for differentiation process. The culture media

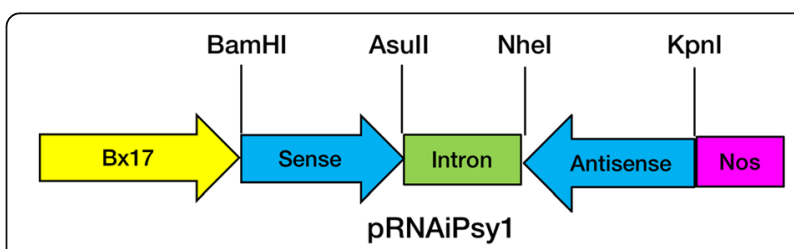

Fig. 1 Non-scale diagram of the RNAi cassette in the transformation plasmid pRNAiPsy1. The trigger fragment of Psy 7 was placed in forward (Sense) and reverse (Antisene) orientations separated by the fourth intron of the wheat Psyl gene (Spacer). Restriction sites used in the RNAi vector construction are indicated. Bx17, endosperm-specific promoter; Nos, Agrobacterium tumefaciens nopaline synthase (Nos) terminator 
are shown in Additional file 2: Table S2. All materials used for RNAi were kept at Crop Research Institute, Shandong Academy of Agricultural Sciences.

Regenerated plants were screened using G418. Surviving plants were transferred to soil and grown to maturity under growth chamber conditions of $22 / 16{ }^{\circ} \mathrm{C}$ day/night temperatures, $50-70 \%$ relative humidity, $16 \mathrm{~h}$ photoperiod, and light intensity of $300 \mu \mathrm{mol}$ photons $\mathrm{m}^{-2} \mathrm{~s}^{-1}$. Transformed plants were verified by PCR using specific primer pairs designed for the FAD2 intron, a part of the pSAABx17 vector (Additional file 1: Table S1). Positive transgenic plants were self-pollinated and harvested in the following generations. $T_{3}$ transgenic lines and nontransformed controls were grown under field conditions in Jinan, Shandong province, during the 2013-14 cropping season. Seeds were sown in $2 \mathrm{~m}$ rows with 20 plants per row, $30 \mathrm{~cm}$ between rows and 3 rows per transgenic line. Transformed plants were verified by PCR and tagged at anthesis. Grains for Psy1 expression analysis were collected at 7-day intervals from 7 to 28 days post anthesis (DPA), immediately frozen in liquid nitrogen, and stored at $-80{ }^{\circ} \mathrm{C}$. Mature grains were harvested for YPC assays.

\section{RNA extraction and gene expression analysis}

Total RNA was extracted from grains of $\mathrm{T}_{3}$ transgenic lines and non-transformed controls at different developmental stages using an RNAprep Pure Plant Kit (Tiangen Biotech, Beijing, China), and then treated with DNase I (Qiagen, Valencia, CA, USA), according to the manufacturer's instructions. RNA purity and concentration were measured using a NanoDrop-2000 spectrophotometer (Thermo Scientific, Wilmington, DE, USA). RNA integrity was evaluated on agarose gels. Reverse transcription was performed with $1 \mu \mathrm{g}$ of total RNA using a PrimeScript ${ }^{\mathrm{mx}}$ RT Reagent Kit (Takara Bio Inc., Otsu, Japan) following the manufacturer's recommended protocol.

Quantitative real-time PCR (qRT-PCR) was performed on a Roche LightCycler 480 (Roche Applied Science, Indianapolis, IN, USA) in $20 \mu \mathrm{l}$ reaction mixtures containing $10 \mu \mathrm{l}$ of LightCycler FastStart DNA Master SYBR Green (Roche Applied Sciences), $0.4 \mu \mathrm{M}$ of each primer, $50 \mathrm{ng}$ of cDNA and $8.2 \mu \mathrm{l}$ of $\mathrm{dd}_{2} \mathrm{O}$. Amplification conditions were an initial $95^{\circ} \mathrm{C}$ for $10 \mathrm{~min}$, and 40 cycles of $95{ }^{\circ} \mathrm{C}$ for $15 \mathrm{~s}, 60{ }^{\circ} \mathrm{C}$ for $20 \mathrm{~s}$ and $72{ }^{\circ} \mathrm{C}$ for $20 \mathrm{~s}$. Fluorescence was acquired at $60{ }^{\circ} \mathrm{C}$. Designs for gene-specific primer amplifying all three Psy1 genes were based on conserved regions among the A, B and D subgenomes. Expression of a $\beta$-actin gene was used as an endogenous control to normalize expression levels of different samples. The primers are listed in Additional file 3: Table S3. Specificities of primers were confirmed by sequencing qRT-PCR products and melt curve analyses. Gene expression levels were presented as multiples of actin levels calculated by the formula $2^{-\Delta \mathrm{CT}}[\Delta \mathrm{CT}=(\mathrm{Ct}$ value of target gene) - (Ct value of actin)] to correct for differential cDNA concentrations among samples [20]. For each line, three biological replicates, each with three technical replicates, were performed and the data were expressed as means \pm standard error (SE).

\section{Yellow pigment content (YPC) assay}

Grains from individual plants of $T_{3}$ transgenic lines and non-transformed controls were ground into whole-grain flour by a Cyclotec ${ }^{\text {tu }} 1093$ mill (Foss Tecator Co., Hillerod, Denmark). The whole-grain flour $(0.5 \mathrm{~g})$ was used for YPC assay following Zhai et al. [21]. Three biological repeats were performed for each line, and each sample was assayed in duplicate; all differences between two repeats were less than $10 \%$.

\section{Transcriptome library construction and RNA sequencing}

To investigate the complex transcriptional regulation network underlying Psy1 down-regulation, deep-sequencing analysis of transcriptomes of transgenic lines and nontransformed controls was performed by RNA-Seq. Three transgenic lines (275-3A, 273-2A and 279-1A) with the most significantly reduced YPC were selected (Fig. 2). Grains of transgenic lines and controls at 14 DPA were used for transcriptome analysis, because this developmental stage showed substantially decreased Psy1 expression (Fig. 3). Total RNA were extracted from pooled grains of six biological repeats per transgenic line or controls and sent to BGI (Beijing Genomics Institute, Shenzhen, China) for RNA-Seq. Transcriptome libraries were prepared and sequenced on the Illumina HiSeq 2000 platform (Illumina, San Diego, CA, USA) following Zhou et al. [22].

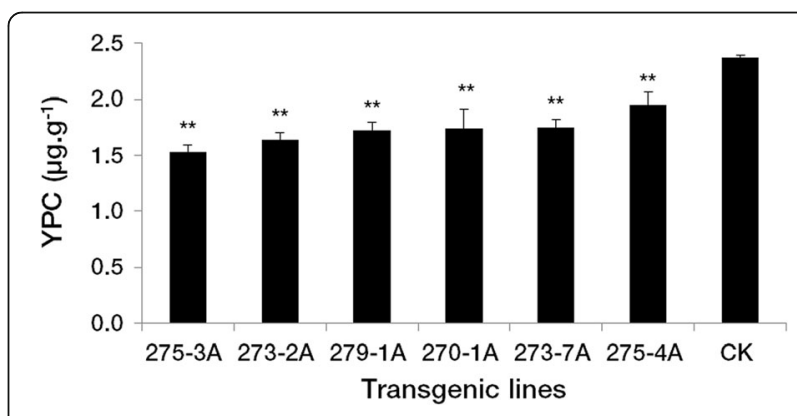

Fig. 2 Yellow pigment content in grains from $T_{3}$ transgenic lines and non-transformed controls. Data are presented as means \pm standard error from three biological replicates. The double asterisks indicate significant differences between transgenic lines and controls at $P=0.01$. CK, non-transformed controls 


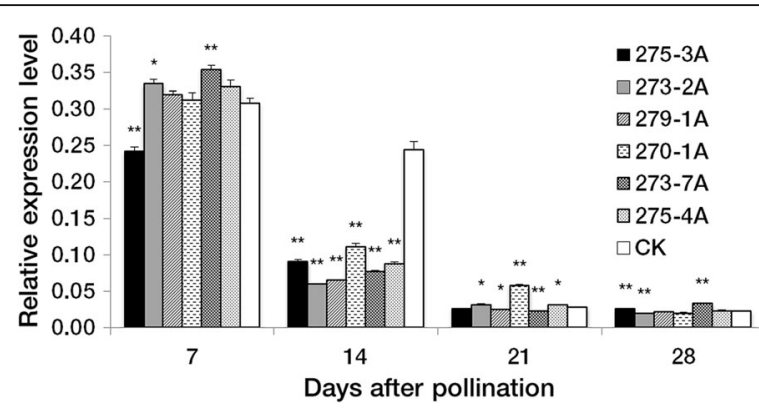

Fig. 3 Expression levels of Psy 1 in developing grains from $T_{3}$ transgenic lines and non-transformed controls. Gene expression levels were measured by qRT-PCR and normalized to the transcript level of a constitutively expressed $\beta$-actin gene in the same sample. Data are presented as means \pm standard error from three biological replicates with three technical replicates each. Significant differences (Student's $t$ test) in transgenic lines compared to the controls are represented by one or two asterisks: ${ }^{*} P<0.05$, ${ }^{* *} P<0.01$. CK, non-transformed controls

\section{Screening and analysis of differentially expressed genes (DEGs)}

Original image data were transformed into sequence data by base calling, and defined as raw reads. Before data analysis, it was prerequisite to remove dirty raw reads including reads with adaptors, those with more than $10 \%$ of unknown bases and low quality reads (more than $50 \%$ low quality bases). Clean reads were then aligned to the reference genome of $T$. aestivum (ftp://ftp.ensemblgenomes.org/pub/plants/release-26/fast a/triticum_aestivum/). Briefly, the clean reads were mapped to the genome reference by BWA software [23] and to the gene reference with Bowtie software [24]. Reads mapping to unique sequences, designated as unigenes, were the most critical subset in the transcriptome libraries as they explicitly identify a transcript. Unigene function was annotated by alignment of the unigenes with the NCBI (National Center for Biotechnology Information) non-redundant $(\mathrm{Nr})$ database using Blastx at an E-value threshold of $10^{-5}$.

Gene expression level was normalized as the FPKM (fragments per kb per million reads) by a RSEM software package [25]. The fold-change in expression of each gene between the transgenic line and non-transformed control was evaluated by FPKM ratio. We used a false discovery rate (FDR) of $<0.001$ and the absolute value of $\mid \log _{2}$ Ratio $\mid \geq 1$ as the threshold to judge the DEGs. To obtain robust and reliable effects of Psy1 down-regulation on gene transcription, only DEGs consistent across all three transgenic lines were chosen for subsequent analysis. Gene ontology (GO) annotation was conducted using the Blast2GO program (https://www.blast2go.com/). The GO categorizations were displayed as three hierarchies, namely biological process (BP), cellular component (CC) and molecular function (MF) by WEGO software [26]. DEGs were also analyzed against the KEGG database (Kyoto Encyclopedia of Genes and Genomes; http://www.genome.jp/kegg/) to explore the potential metabolic pathways that might be involved in reduction of carotenoid synthesis in transgenic lines.

\section{Subcellular localization of PSY1 in wheat}

To investigate subcellular localization of PSY1, the cDNA sequence of Psy1 without the termination codon was isolated from common wheat cultivar Jimai 22 (developed by the Crop Research Institute, Shandong Academy of Agricultural Sciences) using primers, Psy1GFP-F (5'-GCCCAGATCAACTAGTATGGCCACCAC CGTCACGCTGC-3') and Psy1-GFP-R (5'-TCGAGAC GTCTCTAGAGGTCTGGTTATTTCTCAGTG-3'), and confirmed by sequencing. The cDNA of Psy1 was then C-terminally fused to the green fluorescent protein (GFP) gene in the pAN580 vector to create Psy1-GFP under the control of the cauliflower mosaic virus (CaMV) 35S promoter. The Psy1-GFP fusion and GFP were transiently transformed into wheat protoplasts following Zhang et al. [27]. Briefly, the stem and sheath of 30 wheat seedlings were cut into approximately $0.5 \mathrm{~mm}$ strips, which were immediately transferred into $0.6 \mathrm{M}$ mannitol for $10 \mathrm{~min}$ in the dark. After discarding the mannitol, the strips were incubated in an enzyme solution for $4-5 \mathrm{~h}$ in the dark with gentle shaking (60$80 \mathrm{rpm})$. Then, an equal volume of W5 solution was added, followed by vigorous shaking by hand for $10 \mathrm{~s}$. Protoplasts were released by filtering through $40 \mu \mathrm{m}$ nylon meshes into round bottom tubes with $3-5$ washes of the strips using W5 solution. The pellets were collected by centrifugation at 1,500 rpm for $3 \mathrm{~min}$, and were then resuspended in MMG solution. Then, PEGmediated transfections were carried out [28]. Fluorescence images were observed by a Zeiss LSM710 confocal laser microscope (Carl Zeiss MicroImaging GmbH, Germany).

\section{EMS mutagenesis}

Two EMS-mutagenised common wheat populations were constructed following Slade et al. [17] with minor modifications. In brief, approximately 5,000 seeds of common wheat cultivars Jimai 22 and Jimai 20 (developed by the Crop Research Institute, Shandong Academy of Agricultural Sciences) were treated overnight with $1.2 \%$ EMS solution and surviving plants were grown to maturity. Seeds from the leading spikes of the $M_{1}$ plants were harvested and one grain from each plant was sown to generate the $\mathrm{M}_{2}$ population (Jimai 20: 1,250 lines; Jimai 22: 1,240 lines). Genomic DNA was isolated from individual $\mathrm{M}_{2}$ plants for TILLING analysis. Twenty seeds from each $\mathrm{M}_{2}$ line containing a mutation in the Psyl gene and wild type were grown under field conditions for further analysis. 


\section{Mutation screening by TILLING}

DNA samples were extracted from individual $M_{2}$ plants of EMS-mutagenised populations derived from Jimai 20 and Jimai 22. DNA concentration was measured by a NanoDrop-2000 spectrophotometer (Thermo Scientific) and standardized. Equal amounts of DNA from individual plant samples were pooled eightfold and organized into 96-well plates. The optimal target region for TILLING screening, considered as one of the most promising for identifying mutations affecting protein function, was defined by the program CODDLE (Codons Optimized to Discover Deleterious Lesions; http://blocks.fhcrc.org/proweb/coddle/). In conjunction with the CODDLE results, homoeolog-specific primers were designed taking advantage of polymorphisms among the three homoeologs of Psy1 in the hexaploid genome (Additional file 4: Table S4). Primer specificities were validated using Chinese Spring nulli-tetrasomic lines and by sequencing.

A fast and cost-effective method, mismatch-specific endonuclease digestion of heteroduplexes followed by non-denaturing polyacrylamide gels stained with silver, was used for mutation detection, which has similar sensitivity to traditional LI-COR screens [29]. Once a positive individual was found, the amplified product was sequenced to determine the accuracy of the mutation.

PARSESNP (Project Aligned Related Sequences and Evaluate SNPs; http://blocks.fhcrc.org/proweb/parsesnp/ ) was used to indicate the nature of each mutation. The PARSESNP and SIFT (Sorting Intolerant from Tolerant; http://sift.bii.a-star.edu.sg/) programs were used to predict the severity of each mutation. Mutations are predicted to have a severe effect on protein function if PSSM scores are $>10$ and SIFT scores are $<0.05[30,31]$.

\section{Creation and characterization of $\mathrm{F}_{2}$ populations}

To determine the impact of new Psy1 alleles on protein function, homozygous $M_{3}$ mutants carrying non-silent (including truncation and missense) mutations were backcrossed to corresponding wild type plants (Jimai 20 or Jimai 22) to reduce background noise. $F_{1}$ plants were self-pollinated and harvested separately. Two hundred $F_{2}$ seeds from each backcross and wild type were grown under field conditions in Beijing during the 2013-14 cropping season, arranged in a randomized complete block design. Seeds were sown in $2 \mathrm{~m}$ rows with 20 plants per row, $30 \mathrm{~cm}$ between rows and 10 rows per $F_{2}$ population. Three genotypes (homozygous mutant, heterozygous mutant and wild-type genotype) in each $F_{2}$ population were selected by sequencing. Spikes of five biological replicates for each genotype were tagged at anthesis. Immature grains were collected at 7-day intervals from 7 to 28 DPA for Psy1 expression analysis. Mature grains were harvested for YPC assays. All $F_{2}$ populations were conserved at the Crop Germplasm
Resources Conservation Center, Chinese Academy of Agricultural Sciences.

The impacts of new Psy1 alleles on YPC were assessed by comparing the differences between homozygous and heterozygous mutants with wild-type genotypes in each $\mathrm{F}_{2}$ population. YPC was measured by the method described above. All measurements were based on five biological repeats. Wild-type genotypes in each $F_{2}$ population were designated as the calibrator with its value set to 1 . The data are presented as means $\pm \mathrm{SE}$.

qRT-PCR was performed on cDNA from developing grains of each genotype in each $F_{2}$ population at 7,14 , 21 and 28 DPA to investigate the effect of mutations on the expression pattern of the particular Psy1 gene and its homoeologs. Briefly, total RNA was extracted from pooled grains of five biological repeats per genotype. Two sets of primers were designed by comparing coding regions of the three Psy1 homoeologs. The first set of primers amplifying all three homoeologs was used to examine gene-specific expression. The second set, the homoeolog-specific primers, was used to determine expression levels of each homoeolog (Additional file 3: Table S3). The specificity of these primers was tested as described above. The protocol for qRT-PCR was also the same. For each sample three technical replicates were performed. Relative expression was calculated using the $2^{-\Delta \Delta C T}$ method [20]. Relative expression levels of Psy1 and its homoeologs were normalized firstly to the transcript level of $\beta$-actin gene in the same sample and then calculated relative to the value of wild-type genotypes at $28 \mathrm{DPA}$ (set to 1 ) in each $\mathrm{F}_{2}$ population. Expression analysis was performed only on $\mathrm{F}_{2}$ populations for the mutants with significant phenotypic changes.

\section{Functional domains and structural modeling of wheat PSY1}

Functional domains of PSY1 protein were predicted by the NCBI's Conserved Domain Database (CDD; http:// www.ncbi.nlm.nih.gov/Structure/cdd/cdd.shtml). To understand the effect of new Psy1 alleles on protein structure, the three-dimensional structure of PSY1 was generated by the SWISS-MODEL (http://swissmodel.expasy.org/) and visualized using Swiss-PdbViewer (http://www.expasy.org/spdbv/).

\section{Detection of alternative splicing variants}

Splice junction mutations are speculated to have severe effects on protein function because they can lead to aberrant RNA splicing and subsequently altered or truncated protein translation [32]. Although no splice junction mutation was identified in this study, mutation sites in M090122 and M092201 were adjacent to the splice site. The mutation site in M090122 was localized at the 3' end of exon II and that in M092201 was at the second nucleotide from the 3' end of exon V. Reverse transcription PCR 
was performed to investigate whether these mutations led to alternative splicing. Briefly, total RNA were extracted from homozygous mutant and wild type individuals, and reverse transcribed into cDNA by the method described above. The cDNA were amplified using the corresponding primers (Additional file 5: Table S5), and PCR products were analyzed by gel electrophoresis and sequenced.

\section{Statistical analysis}

Data are presented as means \pm SE. Student's $t$ test was used to assess the statistical significance of differences in pairwise comparisons of transgenic lines and nontransformed controls, or between homozygous or heterozygous mutants and wild-type genotypes in each $\mathrm{F}_{2}$ population.

\section{Results}

Psy1 gene expression and YPC in grains of transgenic lines

The 460 bp trigger fragment from $P s y-A 1$ that was used for the RNAi vector construction shared $90 \%$ and $95 \%$ sequence similarity with $P s y-B 1$ and $P s y-D 1$, respectively. Using the Agrobacterium-mediated transformation method six positive, non-segregating transgenic lines, designated as 275-3A, 273-2A, 279-1A, 270-1A, 273-7A and 275-4A, were obtained. They showed no differences in morphology and development compared to non-transformed controls.

The effect of the transformed Psy1-hairpin on Psy1 expression was examined in six positive $\mathrm{T}_{3}$ transgenic lines during grain development. At 7 DPA, qRT-PCR analyses showed a significantly decreased transcript level of Psy1 in the transgenic line 275-3A $(P<0.01)$, significantly increased transcription levels in 273-2A and 273-7A ( $P<0.05$ and $P<0.01$, respectively), and slight changes in the other lines, compared to non-transformed controls. Substantially decreased Psy1 expression levels of $54-76 \%$ were found in all transgenic lines at 14 DPA $(P<0.01)$. At 21 and 28 DPA differences in expression levels between the transgenic lines and controls were very small (2-15\%), except for line 270-1A at 21 DPA and line 273-7A at 28 DPA (Fig. 3). Significantly decreased YPC ranging from 26 to $35 \%$ occurred in all transgenic lines compared with non-transformed controls (Fig. 2).

Transcriptional profiling underlying Psy1 down-regulation Totals of 1,128,107, 1,160,285, 1,192,915 and 1,228,928 unigenes were obtained for transgenic lines 273-2A, 275-3A, 279-1A and the control, respectively (Additional file 6: Table S6). Comparison of the transcript abundances between transgenic lines and controls identified 948, 930 and 992 DEGs for 273-2A, 275-3A and 2791A, respectively (Additional file 6: Table S6). In total, 287 DEGs were consistent across all three transgenic lines, perhaps representing the reliable effects of Psy1 down-regulation on gene transcription (Additional file 7: Table S7).

Categorization of GO terms of the 287 DEGs is shown in Fig. 4. Metabolic process and cellular process were the major categories annotated to the biological process (BP); cell part and cell were the major categories annotated to the cellular component (CC); and catalytic activity and binding were the major categories annotated to the molecular function (MF). Through pathway enrichment analysis, 199 of the 287 DEGs were assigned to 46 metabolic pathways (data not shown). The pathways significantly associated with Psy1 down-regulation included carotenoid biosynthesis, diterpenoid biosynthesis, various types of $\mathrm{N}$-glycan biosynthesis, ubiquinone and other terpenoid-quinone, glycolysis/gluconeogenesis, starch and sucrose metabolism, fructose and mannose metabolism and citrate cycle, photosynthesis, and carbon fixation in photosynthetic organisms (Fig. 5). All candidate genes in relevant pathways are listed in Additional file 8: Table S8.

\section{PSY1 subcellular localization}

Psy1-GFP was constructed and transiently expressed in wheat protoplasts to investigate PSY1 subcellular localization. Protoplasts allow us to observe the localization of transiently

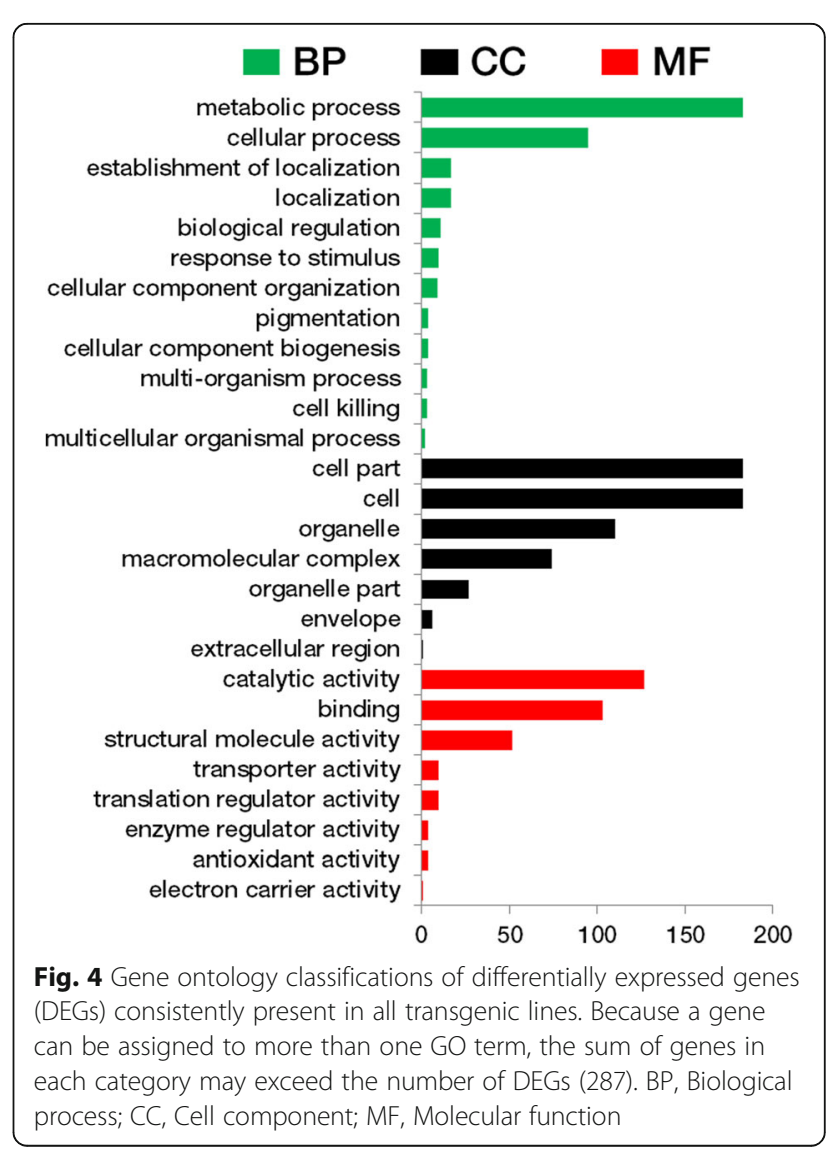




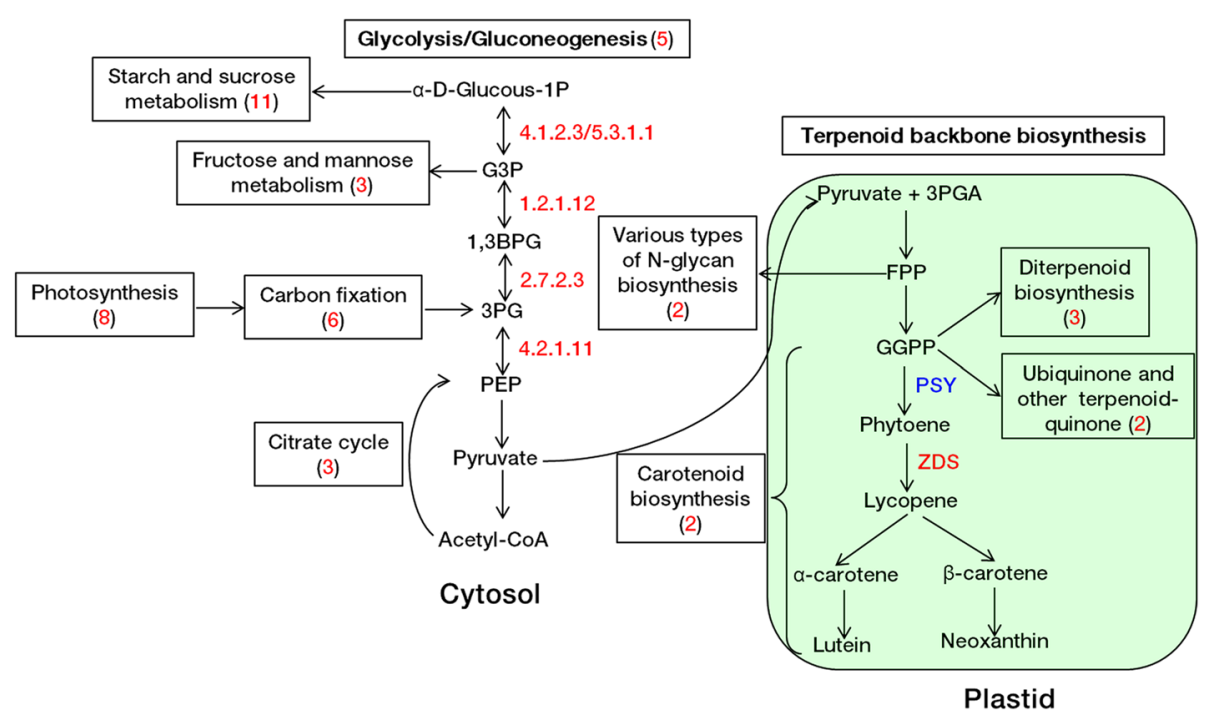

Fig. 5 Overview of major metabolic pathways associated with Psy1 down-regulation in transgenic lines. Genes that were 2-fold greater up- or down-regulated are shown in red or blue, respectively. The number of candidate genes in a relevant pathway is indicated in brackets, and the detail of candidate genes in each pathway is listed in Table S7. 1,3BPG, 3-phospho-D-glyceroyl phosphate; 3PG, 3-phospho-D-glycerate; FPP, farnesyl diphosphate; G3P, glyceraldehyde 3-phosphate; GGPP, geranylgeranyl pyrophosphate; PEP, phosphoenolpyruvate; PSY, phytoene synthase; ZDS, zeta-carotene desaturase

expressed PSY1 proteins, due to retain their tissue specificity after isolation and thereby reflect in vivo conditions. GFP alone was distributed evenly in the cytoplasm and nuclei (data not shown), whereas the Psy1-GFP fusion proteins colocalized exclusively with autofluorescence signals of chlorophyll, indicating that PSY1 was localized in plastids (Fig. 6).

\section{Identification of mutations in Psy 1 by TILLING}

Eighty two new Psy1 alleles were identified in the two EMS-mutagenised populations, including three truncation, 26 missense and 53 silent mutations (Table 1; Additional file 9: Table S9). As expected for alkylation of guanine by EMS, the majority of mutations were $\mathrm{G}$ to $\mathrm{A}$ (61.0 \%) or $\mathrm{C}$ to $\mathrm{T}(31.7 \%)$ transitions, with the exception of six mutations as follows: A to C (2), A to G, A to $\mathrm{T}, \mathrm{T}$ to $\mathrm{C}$ and $\mathrm{T}$ to $\mathrm{G}$.

Two missense mutations (M090628 and M091151) and three truncation mutations (M090158, M090950 and M091949) were predicted to have severe effects on protein function based on SIFT score and PSSM values (Table 2).

\section{Characterization of new alleles of Psy 1}

Twenty-nine $F_{2}$ populations were developed from homozygous $\mathrm{M}_{3}$ mutants carrying non-silent (missense and truncation) mutations and corresponding wild type plants, and YPC assays were carried out to characterize the effects of the non-silent mutations on protein function. As shown in Fig. 7 mutations in Psy-A1, namely M090158, M090950, M091949, M090122 and M091151, significantly reduced YPC by 9-29\% (between homozygous mutants and wild-type sibs), whereas the mutation in Psy-D1 of M091217 significantly increased YPC by $34 \%$.

The expression profiles of Psy1 and its homoeologs in grains of each genotype in the six $F_{2}$ populations were

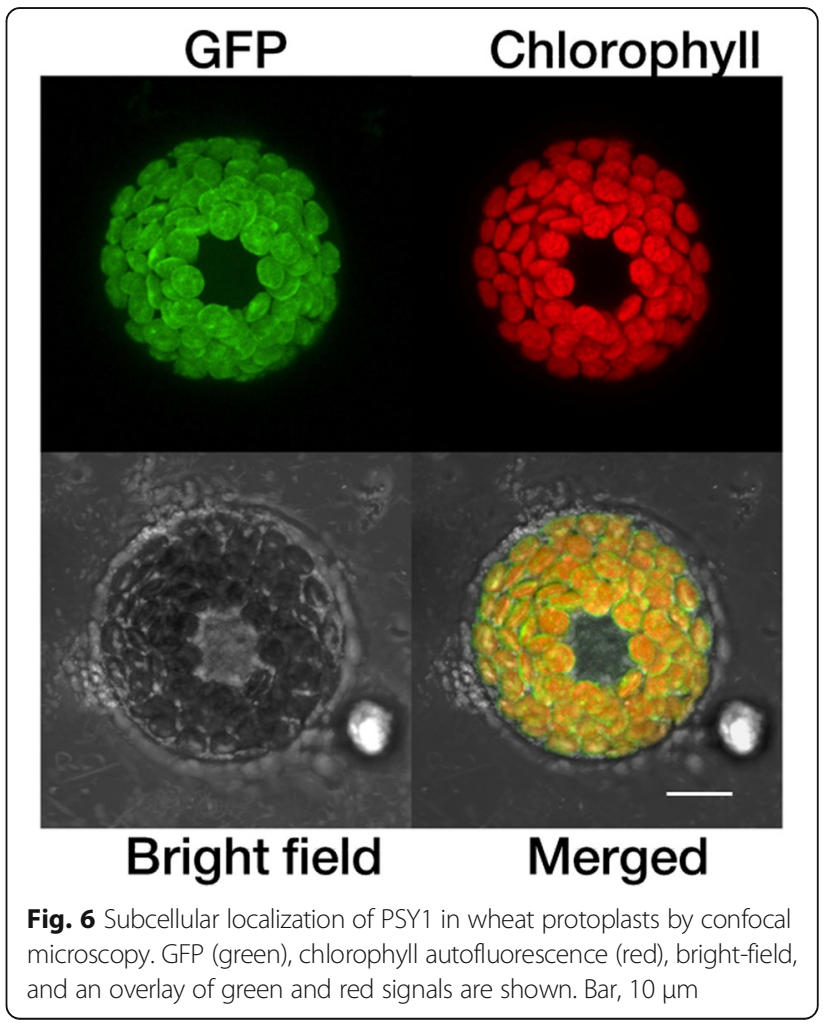


Table 1 Summary of non-silent mutations in Psy1 identified by TILLING

\begin{tabular}{|c|c|c|c|c|c|c|c|}
\hline Gene & M3 Plant & Cultivar & Exon\Vntron & Nucleotide change $^{a}$ & Amino acid change ${ }^{b}$ & Codon change & Zygosityc \\
\hline \multirow[t]{18}{*}{$\overline{P_{S y}-A 1}$} & M091753 & $J 22$ & Exon & C308T & A103V & $\mathrm{GCA} \rightarrow \mathrm{GTA}$ & Hom \\
\hline & M090158 & $J 20$ & Exon & C3201T & Q346* & $\mathrm{CAG} \rightarrow \mathrm{TAG}$ & Hom \\
\hline & M092432 & $J 20$ & Exon & C3255T & L364F & $\mathrm{CTT} \rightarrow \mathrm{TT}$ & Hom \\
\hline & M091887 & $J 22$ & Exon & C335T & S112L & $\mathrm{TCG} \rightarrow \mathrm{TTG}$ & Hom \\
\hline & M091949 & $J 22$ & Exon & С $349 \mathrm{~T}$ & Q117* & $\mathrm{CAG} \rightarrow \mathrm{TAG}$ & Hom \\
\hline & M090950 & $J 22$ & Exon & G1224A & W172* & $\mathrm{TGG} \rightarrow \mathrm{TAG}$ & Hom \\
\hline & M091151 & $J 22$ & Exon & G1230A & R174K & $\mathrm{AGG} \rightarrow \mathrm{AAG}$ & Hom \\
\hline & M090997 & $J 22$ & Exon & G271A & E91K & $\mathrm{GAG} \rightarrow \mathrm{AAG}$ & Hom \\
\hline & M092152 & $J 22$ & Exon & G3231A & E356K & $\mathrm{GAG} \rightarrow \mathrm{AAG}$ & Het \\
\hline & M091102 & $J 22$ & Exon & G3554A & R397K & $\mathrm{AGG} \rightarrow \mathrm{AAG}$ & Hom \\
\hline & M090333 & $J 20$ & Exon & G3605A & G414E & $\mathrm{GGG} \rightarrow \mathrm{GAG}$ & Het \\
\hline & M092889 & $J 22$ & Exon & G371A & $\mathrm{R} 124 \mathrm{~K}$ & $\mathrm{AGG} \rightarrow \mathrm{AAG}$ & Hom \\
\hline & M090755 & $J 20$ & Exon & G400A & G134R & $\mathrm{GGG} \rightarrow \mathrm{AGG}$ & Hom \\
\hline & M092383 & $J 20$ & Exon & G412A & A138T & $\mathrm{GCC} \rightarrow \mathrm{ACC}$ & Het \\
\hline & M091295 & $J 22$ & Exon & G436A & E146K & $\mathrm{GAG} \rightarrow \mathrm{AAG}$ & Hom \\
\hline & M092101 & $J 22$ & Exon & G596A & E160K & $\mathrm{GAG} \rightarrow \mathrm{AAG}$ & Hom \\
\hline & M090122 & $J 20$ & Exon & G629A & V171G & $\mathrm{GTA} \rightarrow \mathrm{AGT}$ & Hom \\
\hline & M092853 & $J 22$ & Exon & T3169G & V335G & $\mathrm{GTC} \rightarrow \mathrm{GGC}$ & Het \\
\hline$P_{S y-B 1}$ & M091983 & $J 22$ & Exon & G2073A & E244K & $\mathrm{GAG} \rightarrow \mathrm{AAG}$ & Het \\
\hline \multirow[t]{10}{*}{ Psy-D1 } & M092201 & $J 20$ & Exon & С $3792 \mathrm{~T}$ & P370L & $\mathrm{CCG} \rightarrow \mathrm{CTG}$ & Het \\
\hline & M091755 & $J 22$ & Exon & C4109T & P409S & $\mathrm{CCT} \rightarrow \mathrm{TCT}$ & Hom \\
\hline & M090628 & $J 20$ & Exon & С4110T & P409L & $\mathrm{CCT} \rightarrow \mathrm{CTT}$ & Hom \\
\hline & M091169 & $J 22$ & Exon & G1347A & D217N & $\mathrm{GAC} \rightarrow \mathrm{AAC}$ & Het \\
\hline & M091217 & $J 22$ & Exon & G3609A & R309K & $\mathrm{AGA} \rightarrow \mathrm{AAA}$ & Hom \\
\hline & M090649 & $J 20$ & Exon & G3761A & V360M & $\mathrm{GTG} \rightarrow \mathrm{ATG}$ & Hom \\
\hline & M090324 & $J 20$ & Exon & G3779A & E366K & $\mathrm{GAG} \rightarrow \mathrm{AAG}$ & Het \\
\hline & M091365 & $J 22$ & Exon & G4049A & D389N & $\mathrm{GAC} \rightarrow \mathrm{AAC}$ & Het \\
\hline & M092126 & $J 22$ & Exon & G4071A & R396K & $\mathrm{AGG} \rightarrow \mathrm{AAG}$ & Hom \\
\hline & M090608 & $J 20$ & Exon & G4097A & V405M & $\mathrm{GTG} \rightarrow \mathrm{ATG}$ & Het \\
\hline
\end{tabular}

${ }^{a}$ the first letter indicates the wild type nucleotide, the number is its position from the start codon, and the last letter is the mutant nucleotide

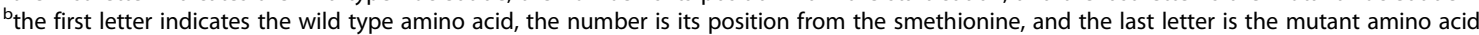

'Hom, homozygous genotype; Het, heterozygous genotype

${ }^{\mathrm{d}}$ bold items, mutations severely affecting phenotype

e*, termination mutation

Table 2 Mutations severely affecting protein function as predicted by the PARSESNP and SIFT programs ${ }^{\mathrm{a}}$

\begin{tabular}{|c|c|c|c|c|c|c|}
\hline Gene & Mutant & Cultivar & Nucleotide change $^{b}$ & Amino acid change $^{c}$ & PSSM & SIFT \\
\hline Psy-A1 & M091151 & $J 22$ & G1230A & R174K & 16.2 & 0.03 \\
\hline Psy-D1 & M090628 & $J 20$ & C4110T & P409L & 18 & 0.04 \\
\hline Psy-A1 & M090158 & $J 20$ & C3201T & Q346* & & \\
\hline Psy-A1 & M090950 & $J 22$ & G1224A & W172* & & \\
\hline Psy-A1 & M091949 & $J 22$ & С349T & Q117* & & \\
\hline
\end{tabular}

${ }^{a}$ High PSSM $(>10)$ and low SIFT scores $(<0.05)$ predict mutations with severe effects on protein function. PSSM and SIFT scores are not reported for mutations that produce premature termination codons

${ }^{\mathrm{b}}$ The first letter indicates the wild type nucleotide, the number is the position from the start codon, and the last letter is the mutant nucleotide

'The first letter indicates the wild type amino acid, the number is the position from the methionine, and the last letter is the mutant amino acid 


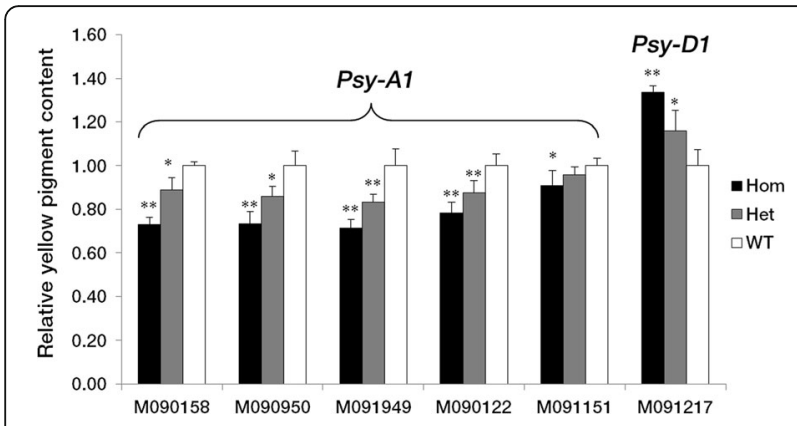

Fig. 7 Relative yellow pigment content of different mutant genotypes in $F_{2}$ populations. $F_{2}$ populations were derived from homozygous nonsilent (truncation and missense) mutants crossed with corresponding controls (Jimai 20 or Jimai 22). Data are given as fold measures relative to wild-type genotypes in each $F_{2}$ population (set to 1). Five biological replicates were performed for each comparison and the data are presented as means \pm standard error. Significant differences (Student's $t$ test) between homozygotes and heterozygotes for the presence of the mutation and wild-type genotypes in each $F_{2}$ population are represented by one or two asterisks: ${ }^{*} P<0.05$, ${ }^{* *} P<0.01$. Hom, homozygous mutants; Het, heterozygous mutants; WT, wild-type genotypes

determined by qRT-PCR at 7, 14, 21 and 28 DPA (Fig. 8). In three populations derived from truncation mutations in Psy-A1 (M090158, M090950 and M091949), Psy-A1 expression levels in homozygous mutants were reduced to $11-48 \%$ compared to wild-type sibs during grain development. Compensatory responses from the $\mathrm{B}$ and $\mathrm{D}$ subgenomes were found to begin at 14 or 21 DPA. For two populations derived from missense mutations in Psy-A1 (M091151 and M090122), the Psy-A1 expression levels in homozygous mutants were more than $33 \%$ of that in wild-type plants, and the compensatory response began at 14 or 28 DPA. For the population derived from the missense mutation in Psy-D1 of M091217, the expression profiles of Psy1 and its homoeologs in homozygous mutants were significantly higher than that of wild-type genotypes during all grain development, except for 21 DPA.

Based on the NCBI's CDD, four characteristic domains were identified in PSY1 protein including aspartate rich regions (DXXXD; substrate- $\mathrm{Mg}^{2+}$-binding sites), a substrate binding pocket, catalytic residues, and active site lid residues (Fig. 9). For three missense mutations significantly influencing YPC and gene expression, the mutation sites of M090122 (V171I) and M091151 (R174K) were adjacent to the ${ }^{177} \mathrm{DXXXD}^{181}$ domain, and the mutation in M091217 (R309K) was close to the ${ }^{302} \mathrm{DXXXD}^{306}$ domain. Threedimensional structure analysis showed that the mutation site of M091217 was located at the entrance of the substrate binding pocket in the PSY-D1 protein (Fig. 10).

\section{Alternative splicing}

The cDNA of grains from homozygous mutants M090122 and M092201 and wild type were amplified and sequenced to investigate the impact of the mutations on pre-mRNA splicing. PCR results for M090122 revealed two products of different size, compared to only the smaller one in wild type individuals (Fig. 11). Sequences of the two transcripts showed that the larger product included a $25 \mathrm{bp}$ fragment of intron II, that resulted in a frame-shift mutation causing a premature termination codon at position 226 (data not shown); the smaller fragment was the constitutive transcript. The M092201 mutant did not produce alternative splicing compared to wild type.

\section{Discussion}

\section{Psy1-specific silencing}

RNAi is a sequence-specific gene suppression system. Previous studies indicated that nucleotide identity between the trigger fragment and target gene is crucial for successful gene silencing by RNAi [33]. It has been suggested that effective gene silencing in higher plants requires $88-100 \%$ nucleotide identity, and $81 \%$ or less nucleotide identities are generally not sufficient for inducing strong and specific gene silencing [34]. In addition, the presence of a continuous stretch of similarity covering at least 21 identical nucleotides between the trigger fragment and target gene is required, although it may not always be sufficient for efficient gene silencing [35, 36]. In this study, the first exon of Psy-A1 (460 bp) was selected as the trigger fragment; it shares $90 \%$ and $95 \%$ nucleotide identity with $P s y-B 1$ and $P s y-D 1$, respectively. Additionally, there were also six contiguous stretches of identical nucleotides longer than $21 \mathrm{nt}$. As expected, all three Psy1 homoeologs were simultaneously silenced, which was proven by RNA-seq (Additional file 7: Table S7).

In grasses, PSY are encoded by three paralogous genes (Psy1-3). The Psy1, Psy2 and Psy3 genes were located to the group 7, 5 and 5 chromosomes, respectively [37]. To determine the gene specificity of our RNAi construct, the sequence similarities among these three genes were analyzed. Psy3 shared $75.4 \%$ nucleotide identity with Psy1 within the $460 \mathrm{bp}$ trigger fragment and had no contiguous stretches of identical nucleotides over $16 \mathrm{nt}$. The sequence of the target region in Psy 2 was not obtained, but the nucleotide identity in the known region was only 74.4 \% compared with Psy1 (data not shown). Therefore, we inferred that the RNAi construct used in the study specifically silenced Ps $y 1$ expression rather than Psy2 and Psy3. In contrast to Psy1, the RNA-seq revealed that the expression levels of Psy2 and Psy3 were not significantly different between transgenic lines and controls (data not shown).

Psy1 expression was not significantly reduced in most transgenic lines at 7 DPA, (Fig. 3), because the $B \times 17$ hardly expresses at this stage [38]. In contrast, Psy1 expression level was substantially decreased in all transgenic 
8AM090158

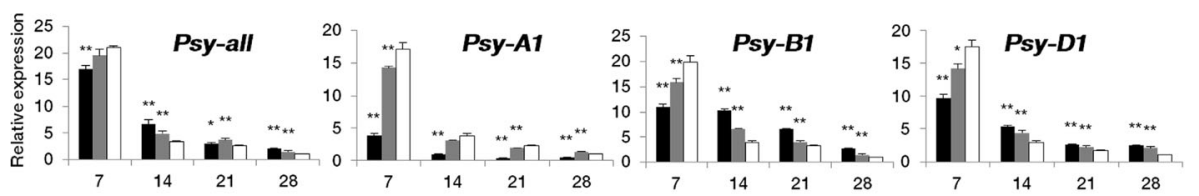

8B M090950

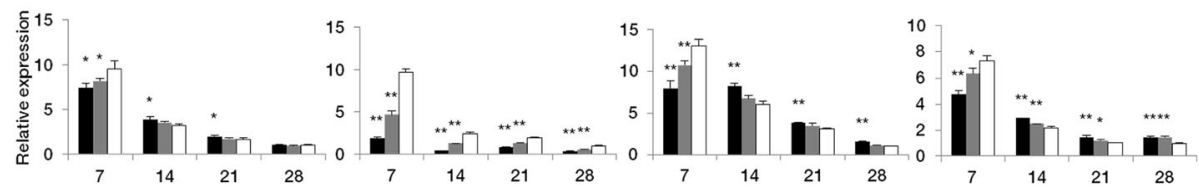

8C M091949

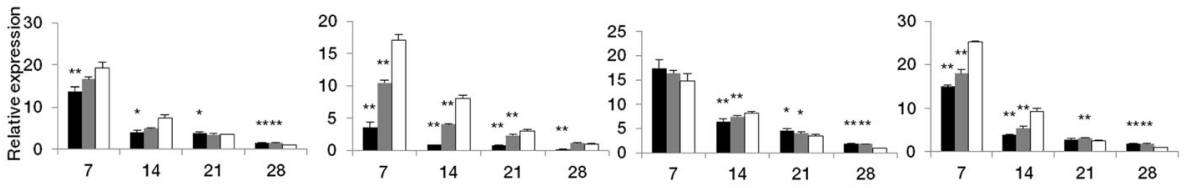

8DM090122

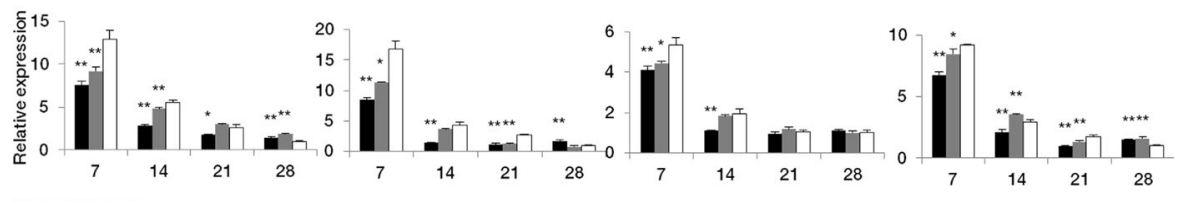

$8 \mathrm{E}$ M091151

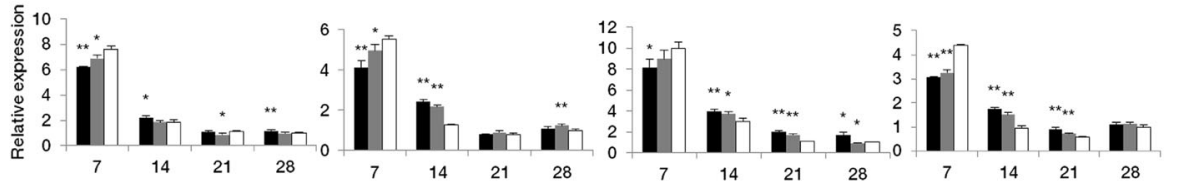

8FM091217

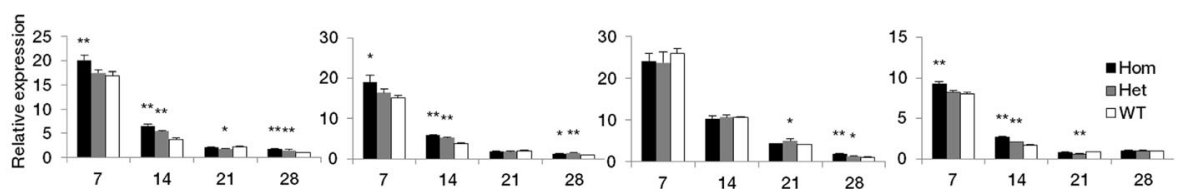

Fig. 8 Expression analysis of Psy1 and its homoeologs in developing grains of three genotypes in each $\mathrm{F}_{2}$ population. a M090158. b M090950. c M091949. d M090122. e M091151. f M01217. For each genotype, five biological repeats were sampled and pooled for RNA extraction and gene expression analysis. Transcript levels are given as expression levels relative to the values of wild-type genotypes at 28 DPA (set to 1 ) after normalization to $\beta$-actin level. Data are presented as means \pm standard error from three technical replicates. Significant differences (Student's $t$ test) between homozygous and heterozygous mutant individuals and wild-type genotypes in each $F_{2}$ population are represented by one or two asterisks: * $P<0.05,{ }^{*} P<0.01$. Hom, homozygous mutants; Het, heterozygous mutants; WT, wild-type genotypes

lines at 14 DPA; this might be attributed to the highest expression level of $B \times 17$ and higher expression of Psy1. In the later developmental stages, the $B x 17$ expression was still very high, whereas Psy1 expression was not reduced distinctly in transgenic lines compared to controls, due to the low expression level of Psy1 and the basic demand of carotenoids for normal growth of plants.

\section{The effect of Psy1 down-regulation}

Quantitative timing analysis of Psy1 expression showed that the RNAi effect was the greatest at 14 DPA, generating 54-76 \% reductions compared to non-transformed controls. As expected, all transgenic lines showed significant YPC reductions, confirming the importance of Psy1 for carotenoid accumulation in wheat grains.

In general, plants have the flexibility to cope with enhancements or reductions of gene products by coordinating the transcriptional regulation network. Pleiotropic effects correlated with up- or down-regulation of Psy genes were reported previously [39], indicating a strong correlation between carotenoid biosynthesis and core metabolism, such as photosynthesis, starch and sucrose metabolism, glycolysis/gluconeogenesis, and the citrate cycle [40-42]. In this study, some candidate genes 


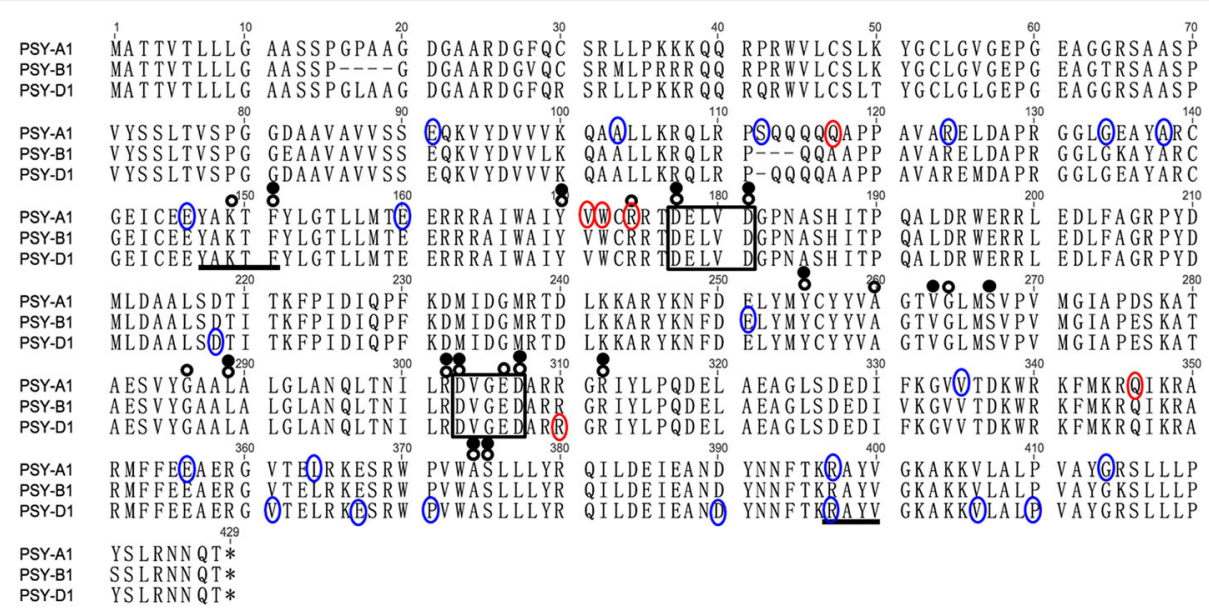

Fig. 9 Functional domains of homoeologous PSY1 protein sequences. Amino acid sequences of PSY1 were analyzed using the NCBI's Conserved Domain Database. Numbers above the alignment indicate the amino acid positions along the PSY-A1 protein. Framed, aspartate rich regions (DXXXD; substrate-Mg ${ }^{2+}$-binding sites); open black circle, substrate binding pocket; filled circle, catalytic residues; line, active site lid residues; blue circle, missense mutations; red circle, mutations resulting in significant yellow pigment content change, including truncation and missense mutations

involved in secondary metabolic pathways and core metabolic processes were found to collectively participate in the adaptive process of Psy1 down-regulation based on RNA-Seq analysis (Fig. 5; Additional file 8: Table S8). In the carotenoid pathway, except for Psy1 down-regulation, up-regulation of the zeta-carotene desaturase gene $(Z d s)$ might be attributed to feedback from reduction of downstream products. Some genes involved in various types of $\mathrm{N}$-glycan biosynthesis, ubiquinone and other terpenoid-quinone biosynthesis and diterpenoid biosynthesis, were up-regulated in transgenic lines. These secondary metabolic pathways compete for FPP (farnesyl diphosphate) or GGPP (geranylgeranyl pyrophosphate) with carotenoid biosynthesis, and therefore carotenoid biosynthesis reduction induces more precursors flow into other pathways. Genes coding enolase (EC 4.2.1.11), phosphoglycerate kinase (EC 2.7.2.3), glyceraldehyde 3-phosphate dehydrogenase (EC

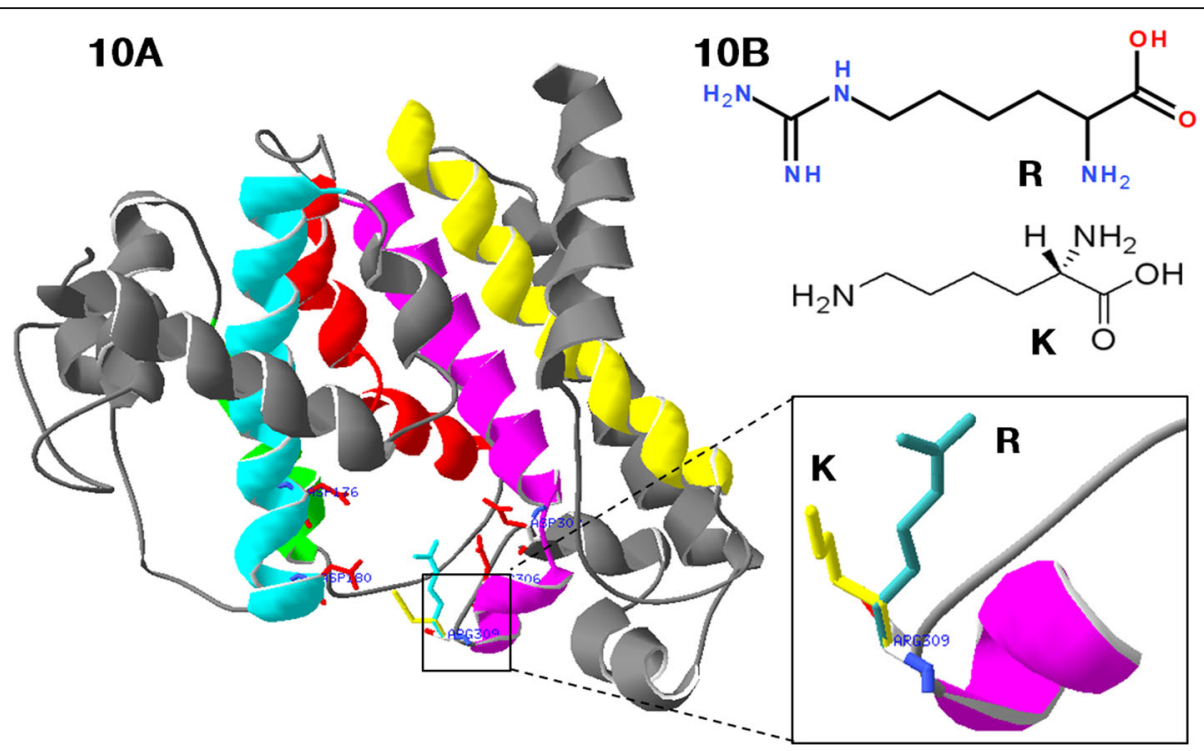

Fig. 10 Graphical representation of PSY-D1 modeled by SWISS-MODEL. a Model of M091217 (R309K) superimposed with wild type. b Carbon skeleton of arginine (R) and lysine (K). The alpha helices at the locations of the substrate binding pocket and catalytic site are shown in bright colors (blue, red, yellow and purple); other helices are in grey. The carbon chain of conserved aspartate in aspartate rich regions (DXXXD) are shown in red, and the carbon chains of $\mathrm{R}$ and $\mathrm{K}$ are in blue and yellow, respectively 


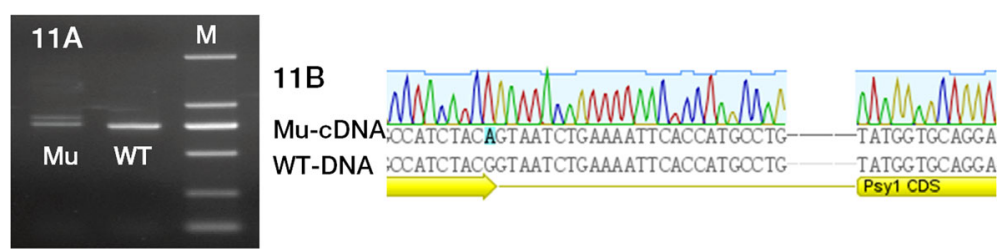

Fig. 11 Alternative splicing in the M090122 mutant. a Reverse transcription PCR analysis showing alternative splicing in M090122. b Alignment of CDNA from homozygous M090122 mutant and DNA sequence of wild type. The sequence traces indicate that the G629A mutation in M090122 caused an alternative splice junction site, located 25 nucleotides downstream of the normal splice junction. Mu, mutagenised line; WT, wild type; M, molecular weight standard DL2000

1.2.1.12), fructose-bisphosphate aldolase (EC 4.1.2.13) and triosephosphate isomerase (EC 5.3.1.1) were upregulated, which might favor the flow into gluconeogenesis since transgenic lines needed a lower flux through and out of the glycolytic pathway for carotenoid biosynthesis. Enhancement of storage reserves synthesis, such as fructose and mannose metabolism and starch and sucrose metabolism, also proved this point. Additionally, enhanced gluconeogenesis further induced photosynthesis, carbon fixation in photosynthetic organisms and citrate cycle. These previously unrecognized YPCrelated-genes in core metabolism established a broader basis for the molecular regulating carotenoid biosynthesis in wheat grains.

\section{Dissection of Psy 1 by TILLING}

TILLING is a flexible strategy for exploring gene function and regulation, producing large series of mutated alleles that may affect protein function and generate partial phenotypic changes or intermediate expression of target genes. In this study, 29 non-silent (truncation and missense) mutations in Psy1 genes in common wheat were identified, providing a resource not only for functional analysis, but also for understanding the importance of different amino acids and regions regulating the protein function, as well as to study compensatory responses.

The severity of each non-silent mutation was predicted by PARSESNP and SIFT, and YPC in each $F_{2}$ population was measured. However, severity prediction was not always consistent with changes in phenotype. For example, the mutation in M090628 was predicted to have a severe effect on protein function, whereas it showed no significant phenotypic change. This might indicate that the conserved sequence had no direct role in controlling enzyme activity, since PARSESNP and SIFT do not account for active or conserved domains, but make predictions based on amino acid conservation and properties after an alignment search in the protein sequence database [30, 31].

Compared with missense mutations in Psy-A1, three truncation mutations showed stronger effects on Psy-A1 expression by reducing Psy-A1 transcript levels in homozygous mutants to $11-48 \%$ of that in wild-type genotypes during whole grain development (Fig. 8). These reductions might be due to a quality control mechanism preventing accumulation of non-functional or deleterious truncated proteins, known as Nonsense Mediated mRNA Decay [43]. In wheat, significantly reduced RNA levels have also been reported for multiple genes containing premature termination codon mutations such as HMW glutenin subunit [44], waxy gene [45], and polyphenol oxidase gene [46].

TILLING is an efficient method to identify mutations in genes of interest, but the mutant effect is often masked by the presence of multiple copies of the same genes in polyploids, such as common wheat. In this study, the expression levels of three homoeologs were measured to study compensatory processes. Unexpectedly, the expression of all three Psy1 homoeologs was significantly reduced or increased together at 7 DPA, except for Psy-B1 in M091949 and M091217 (Fig. 8). In three truncation mutants, the compensatory responses from B and D homoelogs started at 14 DPA for M090158 and M090950 and at 21 DPA for M091949. For missense mutations in M091151 and M090122, the compensatory response began at 14 and 28 DPA, respectively. One possible reason for these phenomena was that the expression of all three Psy1 homoeologs is coordinately regulated under normal conditions, but separately regulated under stress. Furthermore, we inferred that 14 DPA was an important stage for Psy1 expression regulation during wheat grain development because most compensatory responses started at this stage. More detailed investigations are needed to substantiate these hypotheses. Compared with Psy-B1, the expression level of $P s y-A 1$ and $P s y-D 1$ showed more distinct changes, and it seems that they were more sensitive to expression regulation. RNA-seq data also showed that the order of down-regulation level among three homoeologs was $P$ sy-D1 $>$ Psy-A1 $>$ Psy-B1 in transgenic lines (Additional file 7: Table S7).

The nucleotide change (G3609A) in M091217 resulted in substitution of arginine by lysine at position 309 (R309K). 
The three-dimensional structure of PSY1 showed that this mutation was adjacent to the entrance of the substrate binding pocket in the PSY-D1 protein, and was possibly easier for substrate binding due to a shorter carbon chain ( $\mathrm{R}$ to $\mathrm{K}$ ) resulting in increased carotenoid accumulation (Fig. 10). This mutation might coordinately induce expression of all three Psy1 homoeologs, although Psy-B1 showed less changes (Fig. 8). Mutations in gene coding regions have potential to alter plant metabolism in ways other than changing the level of target gene products. For example, a mutated site may change the enzyme-substrate affinity, alter enzyme regulatory domains, or interfere with proper subunit or other protein-protein interactions. The aspartate rich region DXXXD is a conserved domain within isoprenoid synthases and forms an active site to bind phosphate groups of a substrate [47]. In this study, all missense mutants with severe effects on YPC were close to the DXXXD domain, indicating that these regions are very important for PSY1 function. Previous studies showed that sequence variations affecting the catalytic efficiency of the PSY enzyme were as subtle as a single amino acid [48]. Therefore, we infer that these mutations may affect the affinity of PSY1 for phosphate groups of a substrate and further influence carotenoid accumulation.

\section{Alternative splicing}

Sequencing analysis of cDNA indicated that the G629A mutation in M090122 caused an alternative splice junction site, located 25 nucleotides downstream of the normal splice junction (Fig. 11). This mechanism was previously reported in plants and explained by local scanning of the spliceosome to select the best intron splice site based on sequence context [49]. The mutation resulted in a frame shift and a premature termination codon at position 226. We assume that the alternative splicing in M090122 might decrease the content of functional PSY1 protein and further reduce carotenoid biosynthesis. Alternative splicing of Psy1 regulating enzyme activity and carotenoid accumulation was also reported in wheat and Hordeum chilense [50,51].

\section{Molecular breeding}

Mutants identified by TILLING are not involved in genetic modification and can be introduced into breeding programs. The use of mutagenesis in plant breeding is generally considered to have contributed to the release of more than 2,250 crop cultivars with improved yield and quality traits [52]. Therefore, mutants identified in this study will be useful as breeding germplasm for wheat quality improvement. For example, mutants M090158, M090950, M091949 and M090122 with significantly reduced YPC could be used in improvement of wheat genotypes for Chinese style foods such as steamed bread and white Chinese noodles where a bright whiteness is preferred. Meanwhile, M091217 with higher YPC could be useful for improving nutrition because carotenoids are important for human health. Furthermore, these mutants come from elite wheat cultivars Jimai 20 or Jimai 22 and are potentially useful without further pre-breeding to remove undesirable agronomic traits.

\section{Conclusion}

The Psy1 function and genetic regulation in common wheat were extensively analyzed using a complementary reverse genetics approach. The RNAi-mediated downregulation of Psy1 resulted in remarkable reduction in YPC, confirming the important impact of Psy1 on carotenoid accumulation in wheat grains. Based on RNA-Seq and bioinformatics analysis, a series of candidate genes involved in both core metabolic processes and secondary metabolic pathways communicated and worked collaboratively to adapt to the Psy1 down-regulation. The TILLING identified a suite of mutations in Psy1 and provided a more in-depth insight into the gene function, genetic regulation, structure-function relationship, as well as the compensatory response. The aspartate rich region DXXXD, a conserved domain among isoprenoid synthases, was identified as an important region influencing PSY1 function in wheat, and conserved nucleotides adjacent to the domain influenced YPC by regulating gene expression, enzyme activity or alternative splicing. Moreover, the compensatory response played a vital role in gene expression during gain development and 14 DPA was considered as a key regulation node. The findings achieved in the present study would be helpful to further disclose the molecular basis and genetic regulation of carotenoid synthesis in wheat grains and could eventually facilitate the genetic improvement of wheat quality in the future.

\section{Additional files}

Additional file 1: Table S1. Primers used for the RNAi vector construction and positive transgenic line detection. (DOCX $16.7 \mathrm{~kb}$ )

Additional file 2: Table S2. Culture media used in this study for callus induction and differentiation. (DOCX $16.7 \mathrm{~kb}$ )

Additional file 3: Table S3. Primers developed for qRT-PCR analysis. (DOCX $17.5 \mathrm{~kb}$ )

Additional file 4: Table S4. Homoeolog-specific primers developed for mutation detection by TILLING. (DOCX $17.9 \mathrm{~kb}$ )

Additional file 5: Table S5. Primers designed for the detection of alternative splicing. (DOCX $16.6 \mathrm{~kb}$ )

Additional file 6: Table S6. Transcriptome details for three transgenic lines with the most significantly reduced YPC and non-transformed controls. (DOCX $18 \mathrm{~kb}$ )

Additional file 7: Table S7. Details of the differentially expressed genes (DEGs) consistent in all three transgenic lines. (XLSX $82.6 \mathrm{~kb}$ )

Additional file 8: Table S8. Major metabolic pathways and candidate genes associated with Psy1 down-regulation. (XLSX $10.1 \mathrm{~kb}$ )

Additional file 9: Table S9. Summary of silent mutations in Psy 1 identified by TILLING. (XLSX $11.7 \mathrm{~kb}$ ) 


\section{Abbreviations}

DEGs: Differentially expressed genes; DPA: Days post anthesis; EMS: Ethyl methanesulfonate; GFP: Green fluorescent protein; GO: Gene ontology; NCBI: National Center for Biotechnology Information; PARSESNP: Project Aligned Related Sequences and Evaluate SNPS; PSy7: Phytoene synthase 1 ; qRT-PCR: Quantitative real-time PCR; RNAi: RNA interference; RNA-Seq: RNA sequencing; SIFT: Sorting Intolerant from Tolerant; TILLING: Targeting Induced Local Lesions IN Genomes; YPC: Yellow pigment content

\section{Acknowledgements}

We thank Prof. R. A. Mclntosh, Plant Breeding Institute, University of Sydney, for reviewing this manuscript.

\section{Funding}

This work was funded by the National Natural Science Foundation of China (31461143021, 31260327, 31371623), Gene Transformation Projects (2016ZX08009-003, 2016ZX08002003-003), Beijing Municipal Science and Technology Project (D151100004415003), and National Key Project (2016YFD0101802)

\section{Availability of data and materia}

Details of the differentially expressed genes (DEGs) consistent in all three transgenic lines at Additional file 7: Table S7.

Data information for candidate genes and metabolic pathways associated with Psy1 down-regulation at Additional file 8: Table S8.

Data for mutations in Psy 1 identified by TILLING at Table 1 and Additional file 9: Table S9.

\section{Authors' contributions}

SNZ performed the experiment and wrote the paper. GYL, GQS and YLL constructed the RNAi transgenic lines. JMS developed the EMS-mutagenised populations. YWS, JHL and HQL did the field trials. $\mathrm{ZHH}$ and XCX designed the experiment and wrote the paper. All authors read and approved the final manuscript.

\section{Competing interests}

The authors declare that they have no conflict of interest.

\section{Consent for publication}

Not applicable.

\section{Ethics approval and consent to participate}

Not applicable.

\section{Author details}

${ }^{1}$ Institute of Crop Science, National Wheat Improvement Center, Chinese Academy of Agricultural Sciences (CAAS), 12 Zhongguancun South Street, Beijing 100081, China. ${ }^{2}$ Crop Research Institute, Shandong Academy of Agricultural Sciences, 202 Gongye Bei Road, Jinan, Shandong 250100, China. ${ }^{3}$ State Key Laboratory of Plant Cell and Chromosome Engineering, Institute of Genetics and Developmental Biology, Chinese Academy of Sciences, Beijing 100101, China. ${ }^{4}$ International Maize and Wheat Improvement Center (CIMMYT) China Office, c/o CAAS, 12 Zhongguancun South Street, Beijing 100081, China.

Received: 8 July 2016 Accepted: 6 October 2016

Published online: 21 October 2016

\section{References}

1. Cuttriss AJ, Cazzonelli Cl, Wurtzel ET, Pogson BJ. Carotenoids. In: Rébeillé F, Douce R, editors. Biosynthesis of Vitamins in Plants. Amsterdam: Elsevier; 2011. p. 1-36

2. Yeum KJ, Russell RM. Carotenoid bioavailability and bioconversion. Annu Rev Nutr. 2002;22:483-504

3. Harjes CE, Rocheford TR, Bai L, Brutnell TP, Kandianis CB, Sowinski SG, Stapleton AE, Vallabhaneni R, Williams M, Wurtzel ET, Yan JB, Buckler ES. Natural genetic variation in lycopene epsilon cyclase tapped for maize biofortification. Science. 2008;319:330-3.

4. Fraser PD, Bramley PM. The biosynthesis and nutritional uses of carotenoids Prog Lipid Res. 2004;43:228-65.
5. Baublis A, Decker EA, Clydesdale FM. Antioxidant effect of aqueous extracts from wheat based ready-to-eat breakfast cereals. Food Chem. 2000;68:1-6.

6. Zhang W, Dubcovsky J. Association between allelic variation at the phytoene synthase 1 gene and yellow pigment content in the wheat grain. Theor Appl Genet. 2008;116:635-45.

7. Gallagher CE, Matthews PD, Li F, Wurtzel ET. Gene duplication in the carotenoid biosynthetic pathway preceded evolution of the grasses. Plant Physiol. 2004;135:1776-83.

8. Li F, Vallabhaneni R, Wurtzel ET. PSY3, a new member of the phytoene synthase gene family conserved in the Poaceae and regulator of abiotic stress-induced root carotenogenesis. Plant Physiol. 2008;146:1333-45.

9. He XY, Zhang YL, He ZH, Wu YP, Xiao YG, Ma CX, Xia XC. Characterization of phytoene synthase 1 gene (Psy 1 ) located on common wheat chromosome 7A and development of a functional marker. Theor Appl Genet. 2008;116:213-21.

10. Flavell RB, Bennett MD, Smith JB, Smith DB. Genome size and proportion of repeated nucleotide sequence DNA in plants. Biochem Genet. 1974;12:257-69.

11. Lawrence RJ, Pikaard CS. Transgene-induced RNA interference: a strategy for overcoming gene redundancy in polyploids to generate loss-of-function mutations. Plant J. 2003;36:114-21.

12. Travella S, Klimm TE, Keller B. RNA interference-based gene silencing as an efficient tool for functional genomics in hexaploid bread wheat. Plant Physiol. 2006;142:6-20.

13. Sestili F, Janni M, Doherty A, Botticella E, D'Ovidio R, Masci S, Jones HD, Lafiandra D. Increasing the amylose content of durum wheat through silencing of the SBElla genes. BMC Plant Biol. 2010;10:144.

14. Becker D, Wieser $H$, Koehler P, Folck A, Mühling KH, Zörb C. Protein composition and techno-functional properties of transgenic wheat with reduced a-gliadin content obtained by RNA interference. J Appl Bot Food Qual. 2012:85:23.

15. Dong ZY, Feng B, Liang H, Rong CW, Zhang KP, Cao XM, Qin HJ, Liu X, Wang T, Wang DW. Grain-specific reduction in lipoxygenase activity improves flour color quality and seed longevity in common wheat. Mo Breeding. 2015:35:150.

16. Bleeker PM, Spyropoulou EA, Diergaarde PJ, Volpin H, De Both MTJ, Zerbe P Bohlmann J, Falara V, Matsuba Y, Pichersky E, Haring MA, Schuurink RC. RNA-seq discovery, functional characterization, and comparison of sesquiterpene synthases from Solanum lycopersicum and Solanum habrochaites trichomes. Plant Mol Biol. 2011;77:323-36.

17. Slade AJ, Fuerstenberg SI, Loeffler D, Steine MN, Facciotti D. A reverse genetic, nontransgenic approach to wheat crop improvement by TILLING. Nat Biotechnol. 2005;23:75-81.

18. Stemple DL. TILLING-a high-throughput harvest for functional genomics. Nat Rev Genet. 2004:5:145-50.

19. Nadolska-Orczyk A, Przetakiewicz A, Kopera K, Binka A, Orczyk W. Efficient method of Agrobacterium-mediated transformation for triticale (X Triticosecale Wittmack). J. Plant Growth Regul. 2005:24:2-10.

20. Livak KJ, Schmittgen TD. Analysis of relative gene expression data using real-time quantitative $P C R$ and the $2^{-\Delta \Delta C T}$ Method. Methods. 2001:25: 402-8.

21. Zhai SN, He ZH, Wen WE, Jin H, Liu JD, Zhang Y, Liu ZY, Xia XC. Genomewide linkage mapping of flour color-related traits and polyphenol oxidase activity in common wheat. Theor Appl Genet. 2016;129:377-94.

22. Zhou XH, Wang K, Lv DW, Wu CJ, Li JR, Zhao P, Lin ZS, Du LP, Yan YM, Ye XG. Global analysis of differentially expressed genes and proteins in the wheat callus infected by Agrobacterium tumefaciens. PLoS ONE. 2013;8:e79390.

23. Li H, Durbin R. Fast and accurate short read alignment with BurrowsWheeler transform. Bioinformatics. 2009:25:1754-60.

24. Langmead B, Trapnell C, Pop M, Salzber SL. Ultrafast and memory-efficient alignment of short DNA sequences to the human genome. Genome Biol. 2009:10:25-34.

25. Li B, Dewey CN. RSEM: accurate transcript quantification from RNA-Seq data with or without a reference genome. BMC Bioinformatics. 2011:12:323.

26. Ye J, Fang L, Zheng HK, Zhang Y, Chen J, Zhang ZJ, Wang J, Li ST, Li RQ, Bolund L, Wang J. WEGO: a web tool for plotting GO annotations. Nucleic Acids Res. 2006;34:293-7.

27. Zhang Y, Su J, Duan S, Ao Y, Dai JR, Liu J, Wang P, Li YG, Liu B, Feng DR, Wang JF, Wang HB. A highly efficient rice green tissue protoplast system for transient gene expression and studying light/chloroplast-related processes. Plant Methods. 2011;7:30-8. 
28. Yoo SD, Cho YH, Sheen J. Arabidopsis mesophyll protoplasts: a versatile cell system for transient gene expression analysis. Nat Protoc. 2007;2:1565-72.

29. Uauy C, Paraiso F, Colasuonno P, Tran RK, Tsai H, Berardi S, Comai L, Dubcovsky J. A modified TILLING approach to detect induced mutations in tetraploid and hexaploid wheat. BMC Plant Biol. 2009;9:115.

30. Ng PC, Henikoff S. SIFT: predicting amino acid changes that affect protein function. Nucleic Acids Res. 2003;31:3812-4.

31. Taylor NE, Greene EA. PARSESNP: a tool for the analysis of nucleotide polymorphisms. Nucleic Acids Res. 2003;31:3808-11.

32. Slade AJ, McGuire C, Loeffler D, Mullenberg J, Skinner W, Fazio G, Holm A Brandt KM, Steine MN, Goodstal JF. Development of high amylose wheat through TILLING. BMC Plant Biol. 2012;12:69.

33. Wesley SV, Helliwell CA, Smith NA, Wang MB, Rouse DT, Liu Q, Gooding PS, Singh SP, Abbott D, Stoutjesdijk PA, Robinson SP, Gleave AP, Green AG, et al. Construct design for efficient, effective and high-throughput gene silencing in plants. Plant J. 2001;27:581-90.

34. Holzberg S, Brosio P, Gross C, Pogue GP. Barley stripe mosaic virus induced gene silencing in a monocot plant. Plant J. 2002;30:315-27.

35. Miki D, Itoh R, Shimamoto K. RNA silencing of single and multiple members in a gene family of rice. Plant Physiol. 2005;138:1903-13.

36. McGinnis K, Murphy N, Carlson AR, Akula A, Akula C, Basinger H, Carlson M, Hermanson P, Kovacevic N, McGill MA, Seshadri V, Yoyokie J, Cone K, et al. Assessing the efficiency of RNA interference for maize functional genomics. Plant Physiol. 2007;143:1441-51.

37. Dibari B, Murat F, Chosson A, Gautier V, Poncet C, Lecomte P, Mercier I, Bergès $\mathrm{H}$, Pont $\mathrm{C}$, Blanco A, Salse J. Deciphering the genomic structure, function and evolution of carotenogenesis related phytoene synthases in grasses. BMC Genomics. 2012;13:221.

38. Shewry PR, Underwood C, Wan YF, Lovegrove A, Bhandari D, Toole G, Mills ENC, Denyer K, Mitchell RAC. Storage product synthesis and accumulation in developing grains of wheat. J Cereal Sci. 2009;50:106-12.

39. Decourcelle M, Perez-Fons L, Baulande S, Steiger S, Couvelard L, Hem S, Zhu CF, Capell T, Christou P, Fraser P. Combined transcript, proteome, and metabolite analysis of transgenic maize seeds engineered for enhanced carotenoid synthesis reveals pleotropic effects in core metabolism. J Exp Bot. 2015;66:3141-50.

40. Xu Q, Yu KQ, Zhu AD, Ye JL, Liu Q, Zhang JC, Deng XX. Comparative transcripts profiling reveals new insight into molecular processes regulating lycopene accumulation in a sweet orange (Citrus sinensis) red-flesh mutant. BMC Genomics. 2009:10:540.

41. Enfissi EMA, Barneche F, Ahmed I, Lichtlé C, Gerrish C, McQuinn RP, Giovannoni JJ, Lopez-Juez E, Bowler C, Bramley PM. Integrative transcript and metabolite analysis of nutritionally enhanced DE-ETIOLATED1 downregulated tomato fruit. Plant Cell. 2010;22:1190-215.

42. Pan $Z Y$, Zeng $Y L, A n J Y, Y e J L, X u Q$, Deng $X X$. An integrative analysis of transcriptome and proteome provides new insights into carotenoid biosynthesis and regulation in sweet orange fruits. J Proteomics. 2012;75: 2670-84.

43. Baker KE, Parker R. Nonsense-mediated mRNA decay: terminating erroneous gene expression. Curr Opin Cell Biol. 2004;16:293-9.

44. Zhu Y, Li Y, Chen Y, Li H, Liang H, Yue S, Zhang A, Zhang X, Wang D, Jia $X$. Generation and characterization of a high molecular weight glutenin 1Bx14-deficient mutant in common wheat. Plant Breed. 2005; 124:421-7.

45. Saito $M$, Nakamura T. Two point mutations identified in emmer wheat generate null Wx-A1 alleles. Theor Appl Genet. 2005;110:276-82.

46. Sun YW, He ZH, Ma WJ, Xia XC. Alternative splicing in the coding region of Ppo-A1 directly influences the polyphenol oxidase activity in common wheat (Triticum aestivum L.). Funct. Integr. Genomic. 2011;11:85-93.

47. Pandit J, Danley DE, Schulte GK, Mazzalupo S, Pauly TA, Hayward CM, Hamanaka ES, Thompson JF, Harwood Jr HJ. Crystal structure of human squalene synthase A key enzyme in cholesterol biosynthesis. J Biol Chem. 2000:275:30610-7.

48. Welsch R, Arango J, Bär C, Salazar B, Al-Babili S, Beltrán J, Chavarriaga P, Ceballos H, Tohme J, Beyer P. Provitamin A accumulation in cassava (Manihot esculenta) roots driven by a single nucleotide polymorphism in a phytoene synthase gene. Plant Cell. 2010;22:3348-56.

49. Smith CJW, Chu TT, Nadal-Ginard B. Scanning and competition between AGs are involved in 3' splice site selection in mammalian introns. Mol Cell Biol. 1993;13:4939-52.
50. Howitt CA, Cavanagh CR, Bowerman AF, Cazzonelli C, Rampling L, Mimica $J$, Pogson BJ. Alternative splicing, activation of cryptic exons and amino acid substitutions in carotenoid biosynthetic genes are associated with lutein accumulation in wheat endosperm. Funct Integr Genomic. 2009;9: 363-76.

51. Rodríguez-Suárez C, Atienza SG, Pistón F. Allelic variation, alternative splicing and expression analysis of Psyl gene in Hordeum chilense Roem. et Schult. PLoS ONE. 2011;6:e19885.

52. Ahloowalia BS, Maluszynski M, Nichterlein K. Global impact of mutationderived varieties. Euphytica. 2004;135:187-204

\section{Submit your next manuscript to BioMed Central and we will help you at every step:}

- We accept pre-submission inquiries

- Our selector tool helps you to find the most relevant journal

- We provide round the clock customer support

- Convenient online submission

- Thorough peer review

- Inclusion in PubMed and all major indexing services

- Maximum visibility for your research

Submit your manuscript at www.biomedcentral.com/submit
) Biomed Central 